\title{
An Inversion Transformation of Feeding Waveform and Field Distribution on NEMP Simulator
}

\author{
Heng Zhou \\ Northwest Institute of Nuclear Technology, Xi'an, China \\ Email: zhouheng19820510@163.com
}

How to cite this paper: Zhou, H. (2018) An Inversion Transformation of Feeding Waveform and Field Distribution on NEMP Simulator. Journal of Computer and Communications, 6, 210-214.

https://doi.org/10.4236/jcc.2018.612020

Received: November 29, 2018 Accepted: December 27, 2018 Published: December 30, 2018

\begin{abstract}
The paper presents an inverse derivation method for the excitation signal of NEMP simulator and the field distribution near the simulator, and only one field measurement system is needed. Transformation functions from feeding point to arbitrary point have been calculated by the simulator's external shape and the geometrical relationship between simulator and the ground, then with the waveform measured in one point, the excitation signal of NEMP simulator can be obtained, and the field distribution near the simulator can be depicted.
\end{abstract}

\section{Keywords}

Inversion, Fourier, Simulator, NEMP

\section{Introduction}

NEMP simulator is a device which forms NEMP field. Several NEMP simulators had built in last century, to examining the electromagnetic vulnerability of military electronic equipment [1] [2] [3] [4]. The cost of building, operating and preservation these simulators are very expensive, and those simulators are designed by pulsed power technology, which means operating on MV voltage level, and the possibility of breakdown is high correspondingly, therefore breakdown prediction and prevention are especially important for reducing cost and improving efficiency and reliability. There are various techniques to monitor modules' conditions during operation, and collect information for diagnosing abnormity. One of these techniques is measuring the radiation field, which not only for providing diagnose information, but for depicting the field distribution 
near simulator. If the fields of every space point are measured directly, multi field measure systems are required to obtain complete information of field distribution, and the costs are unbearable. Based on the knowledge of linear characteristic of transmission, the paper presents an inverse derivation, by which only one field measure system is required to get field distribution.

In principle, the field near the simulator can be determined by the excitation signal in the feed point of cone and the electromagnetic boundary condition. The electromagnetic boundary condition can be determined by the simulator's external shape and the geometrical relationship between simulator and the ground, the information of which can be obtained by the design drawing of simulator and the simulator's height in a specific experiment. It is hard to measure the excitation voltage signal in the feed point of cone directly, because the voltage signal is about MV level. According to the simulator's boundary condition, the paper calculates the transformation function from the feed point to the point measured, with the data of the measured field signal, the feeding signal on the cone can be calculated. The transformation function from the feed point to other space point can also be calculated by the same way, associate with the feeding signal calculated ahead, the field of other space point can be obtained. Therefore, only one field measure system is required to get field distribution, one can also verify another system's measured data by two field measure system.

\section{Modeling of the Simulator}

In order to calculate the transformation function from the feed point to the point measured, the simulator can be modeled shown as in Figure 1, where the cone feed point can be set as origin point of the coordinate. Several simplifications have been made during modeling: firstly, the electromagnetic boundary conditions in the model are only needed for radiation field transmission, it is not necessary to model the components which generate the voltage pulse; secondly, the switch physical mechanism of the peaking capacity breakdown can be simplified by the discrete port; thirdly, the lines in the cage antenna are dense enough to be considered as the surface of the cylinder, so solid PEC cylinder replace the cage antenna as the electromagnetic boundary condition, the simplification can reduce the size of the model grid number, which increase the calculation efficiency. Fourthly, the spectrum range of NEMP pulse is about $0-200$ $\mathrm{MHz}$, considering the ground roughness, the ground can be set as normal dielectric material with smooth surface and permittivity $\varepsilon_{r}=9$.

The simulator's geometric size along the $Z$ axis is about hundred meters, in order to improve the calculation efficiency, symmetry planes can be set as $H_{t}=0$ on plane $x=0$ and $E t=0$ on plane $z=0$. PML boundary causes the space truncation of the simulator, so Gauss pulse has been employed as the excitation signal for its excellent time local performance, which could depress the deviation of transformation function in low frequency range. Moreover, Gauss pulse with proper parameter can cover the spectrum of NEMP pulse, shown as in Figure 2. 


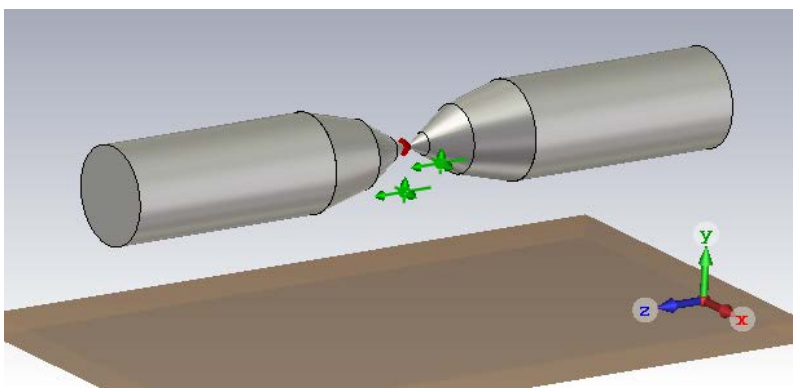

Figure 1. Simulator model and points concerned.

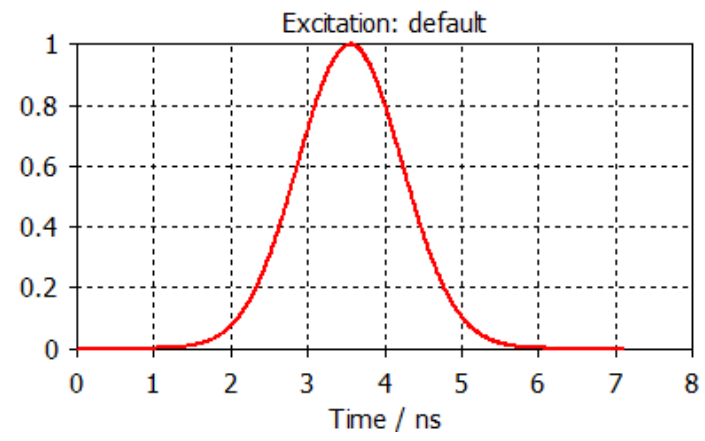

Figure 2. Gauss pulse employed for generation of transformation function.

In order to weaken ground scatter, the point chosen for field measurement should be near the feed point and far from the ground, point $(5,0,0)$ has been chosen in this paper, the length unit is meter. Another point $(3,0,0)$ has been chosen for method verification. The field amplitude in the two chosen point are both in the dynamic range of the field measure system.

\section{Transformation Function and Inversion Derivation}

Transformation function from the feed point to the arbitrary point could be expressed by

$$
H_{r-\text { feed }}(\omega)=F T\left[E_{r}(t)\right] / F T\left[i_{g}(t)\right]
$$

where FT[ $\bullet$ is Fourier transformation, $i_{g}(t)$ is the Gauss excitation signal, $\mathrm{r}$ presents the position. The field signal of every point can be acquired by FIT simulation, according to the equation [1], the transformation function from the feed point to every point could be achieved. For example, the field waveform of point $(5,0,0)$ is shown in Figure 3.

The transformation function $\mathrm{H}(3,0,0)$-feed $(\omega)$ and $\mathrm{H}(5,0,0)$-feed $(\omega)$ could be resolved by equation [1]. Polar coordinate has been employed to show the amplitude and phase of transformation function, shown as in Figure 4.

A numerical simulation has been carried out instead of practical experiment for the technique validation. An NEMP waveform is employed to excite the same simulator model, the field waveform of point $(5,0,0)$ can be obtained like practical measurement, so the excitation waveform of feed point can be deduced by the following equation with the transformation function gained ahead. 


$$
i_{I F T}(t)=\operatorname{IFT}\left[F T\left[E_{(5,0,0)}(t)\right] / H_{(5,0,0)-\text { feed }}(\omega)\right]
$$

where IFT[ $\bullet$ ] is inverse Fourier transformation. The excitation signal il set in the simulation can be regard as the actual excitation signal. The calculated excitation signal i_IFT is identical with i1, shown in Figure 5, which shows the calculated excitation signal can be regarded as the real excitation signal. Meanwhile, the inverse derivation method doesn't depend on the specific form of the excitation signal, the excitation signal's spectrum in the domain of definition is only required.

Furthermore, the waveform of point $(5,0,0)$ can be calculated by

$$
E_{(3,0,0)}(t)=\operatorname{IFT}\left[H_{(3,0,0) \text {-feed }}(\omega) \cdot F T\left[E_{(5,0,0)}(t)\right] / H_{(5,0,0)-\text { feed }}(\omega)\right]
$$

Similarly, the field of point $(3,0,0)$ simulated by FIT arithmetic can be regard as the real field. The deduced waveform of point $(3,0,0)$ reverts the real field waveform precisely including the reflection from the ground scatter, shown in Figure 6.

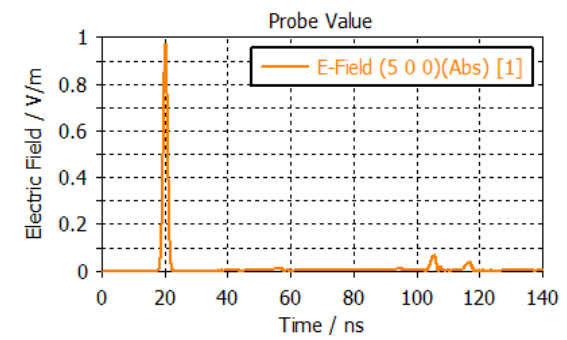

Figure 3. The field waveform of point $(5,0,0)$.

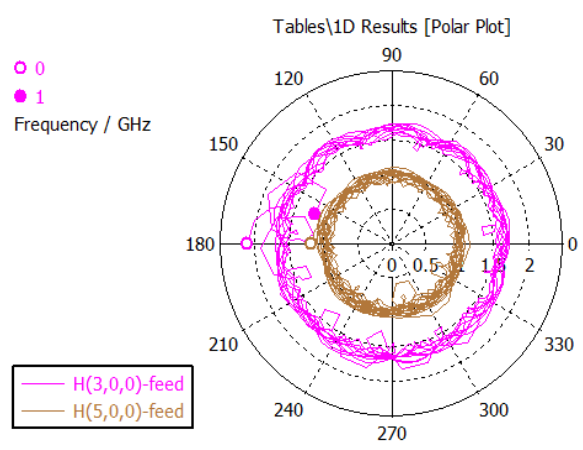

Figure 4. The field waveform of point $(5,0,0)$.

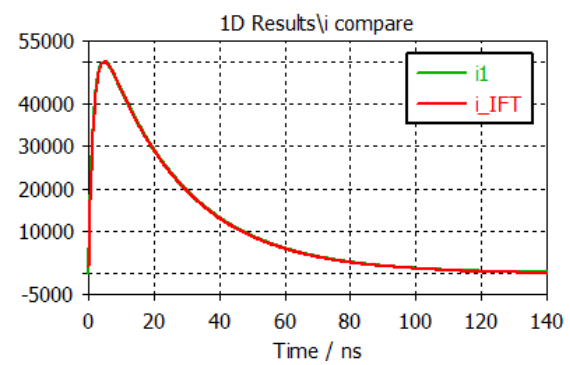

Figure 5. The excitation waveforms of set in the simulation and deduced by inverse transformation. 


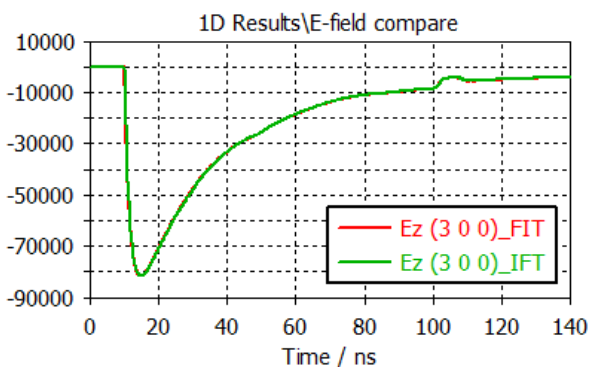

Figure 6. The field waveforms of point $(3,0,0)$ simulated by FIT and deduced by inverse transformation.

\section{Conclusion}

The paper presents an inverse derivation method for the excitation signal of simulator and the field distribution near the simulator, and only one field measurement system is needed. The simulator's external shape and the geometrical relationship between simulator and the ground determine the electromagnetic boundary condition and the transformation function; these transformation functions contain complete information of the transmission in the boundary determined system.

\section{Acknowledgements}

Thanks to Jiang Ting-Yong for giving the opportunity and supports to do the work.

\section{Conflicts of Interest}

The author declares no conflicts of interest regarding the publication of this paper.

\section{References}

[1] Baum, C.E. (1978) Simulators for Various Types of Nuclear EMP Environments. IEEE Transactions on Electromagnetic Compatibility, 20, 35-53. https://doi.org/10.1109/TEMC.1978.303691

[2] Baum, C.E. (1982) Review of Hybrid and Equivalent-Electric-Dipole EMP Simulators. Sensor and Simulation Notes 277.

[3] Robb, J.D. (1986) An Experimental and Theoretical Theoretical Study of Nuclear-EMP-Type Lightning Simulators with Combined Peaking Capacitor and Crowbar. AHWAL-TR-86-3045.

[4] Daniel, K.B. and Tadeusz, W.W. (1988) Concept of an Electromagnetic Pulse Laboratory. Proc. Int. Symp in EMC, Wroclaw Poland, 141-144. 